\title{
NDE in-process for metal parts fabricated using powder based additive manufacturing
}

Leonard Bond, Lucas Koester, Hossein Taheri

Leonard J. Bond, Lucas W. Koester, Hossein Taheri, "NDE in-process for metal parts fabricated using powder based additive manufacturing," Proc. SPIE 10973, Smart Structures and NDE for Energy Systems and Industry 4.0, 1097302 (18 March 2019); doi: 10.1117/12.2520611

Event: SPIE Smart Structures + Nondestructive Evaluation, 2019, Denver, Colorado, United States 


\title{
NDE in-process for metal parts fabricated using powder based
} additive manufacturing

\author{
Leonard J. Bond *a, Lucas W. Koester ${ }^{\mathrm{b}}$ and Hossein Taheric \\ ${ }^{a}$ Department of Aerospace Engineering, Iowa State University, Ames, IA, USA, 50011, \\ ${ }^{\mathrm{b}}$ Centre for NDE, Iowa State University, Ames, IA, USA, 50011, \\ ${ }^{c}$ Department of Manufacturing Engineering, Georgia Southern University, Statesboro, GA 30460
}

\begin{abstract}
Ensuring adequate quality for additive manufactured (AM) materials presents unique metrology challenges to the on-line process measurement and nondestructive evaluation (NDE) communities. AM parts now have complex forms that are not possible using subtractive manufacturing and there are moves for their use in safety criticality components. This paper briefly reviews the status, challenges and metrology opportunities throughout the AM process from powder to finished parts. The primary focus is on new acoustic signatures that have been demonstrated to correlate process parameters with on-line measurement for monitoring and characterization during the build. In-process, quantitative characterization and monitoring of material state is anticipated to be potentially transformational in advancing adoption of metal AM parts, including offering the potential for early part rejection, part condition guided process control or even potentially in-process repair. This approach will enable more effective deployment of quality assessment metrology into the layer-by-layer material build with designed morphology. In this proof-of-concept study acoustic-based process monitoring signals were collected during the Direct Energy Deposition (DED) AM process with different process conditions to investigate and determine if variations in process conditions can be discriminated. A novel application of signal processing tools is used for the identification and use of metrics based on temporal and spectral features in acoustic signals for the purpose of in-situ monitoring and characterization of conditions in an AM process. Results show that the features identified in signatures are correlated with the process conditions and can be used for classifying different states in the process.
\end{abstract}

Keywords: additive manufacturing, in-process monitoring, NDE, acoustic signatures, process condition.

\section{INTRODUCTION}

There are significant challenges faced in fabrication and then the subsequent life cycle of AM parts when seeking to ensure needed initial quality and reliability. The inspection and characterization of powder metal parts at various points in the manufacturing process has been under consideration for several decades, with much of the attention focusing on non-destructive testing (NDT) of finished or near-finished parts ${ }^{1,2}$. Over the years some methods have been demonstrated for application at interim fabrication steps and at other interim points during manufacture ${ }^{3-5}$. Critical to understanding NDT needs is material characterization and "allowables" (those "naturally" occurring material anomalies that are acceptable), such as some level of micro-porosity and grain variation that will not impact performance under some defined set of stressors ${ }^{6}$.

Providing the capability for in-situ monitoring has the advantage of not only inspection of the part to ensure that it is produced to meet the design requirements, but also to detect anomalies which occur within the build process ${ }^{7}$. There are several technologies that are currently deployed, including those using optical ${ }^{8}$ and IR imaging. The advantage of using acoustic and ultrasound-based techniques is that the acoustic signatures have the potential to contain rich information about the material properties, defects and anomalies, as well as the process conditions.

\section{ACOUSTIC IN-SITU PROCESS MONITORING}

Acoustic and ultrasonic-based methods have the advantage of being able to be deployed to give real-time continuous monitoring of a part during the manufacturing processes. Such techniques can be used for process monitoring with different forms of sensors ${ }^{10}$, with laser generated and detected ultrasound ${ }^{11,12}$ and for monitoring the acoustic emissions

*bondlj@iastate.edu; $\quad$ phone 15152941055

Smart Structures and NDE for Energy Systems and Industry 4.0, edited by Norbert G. Meyendorf,

Kerrie Gath, Christopher Niezrecki, Proc. of SPIE Vol. 10973, 1097302 · @ 2019

SPIE · CCC code: $0277-786 \mathrm{X} / 19 / \$ 18 \cdot$ doi: $10.1117 / 12.2520611$ 
from cracking events ${ }^{8}$. Considering the successful application of acoustic-based monitoring techniques for other manufacturing processes, these techniques seem to have the potential for in-situ additive manufacturing process monitoring ${ }^{13,14}$ and some new systems and techniques have been developed for this purpose ${ }^{15}$. For reliable monitoring of the additive manufacturing process, it is important to identify signatures, develop metrics and the transient processrelated signals in the presence of potentially high levels of time-varying noise, generated by the AM machine and processing environment. Both temporal and spectral features can potentially carry useful information that are correlated to the process and part conditions and which can be used for quality monitoring purposes.

\section{Experimental setup and data collection}

An instrumentation system together with an experimental fixture that supports piezoelectric acoustic sensors was designed to enable attachment to the control stage of a Direct Energy Deposition (DED) system. Titanium 6Al-4V powder was deposited on a steel substrate under a variety of conditions. The fixture built to support sensors for process monitoring is shown in Figure 1. The arrangement and dimensions of the specimens and the sensor locations are shown in Figure 2.

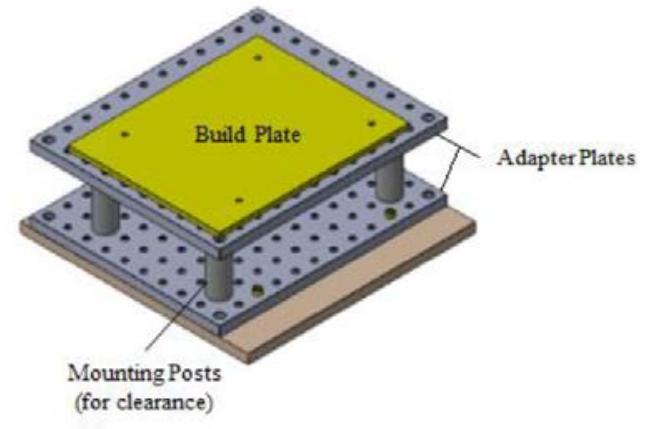

(a)

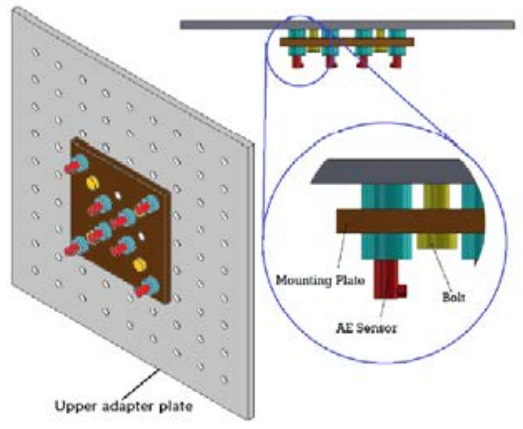

(b)

Figure 1. Monitoring Fixture and accessories, (a) upper and lower adapter plates, build plate and mounting posts, (b) attaching the mounting plate and sensors to the upper adapter plate

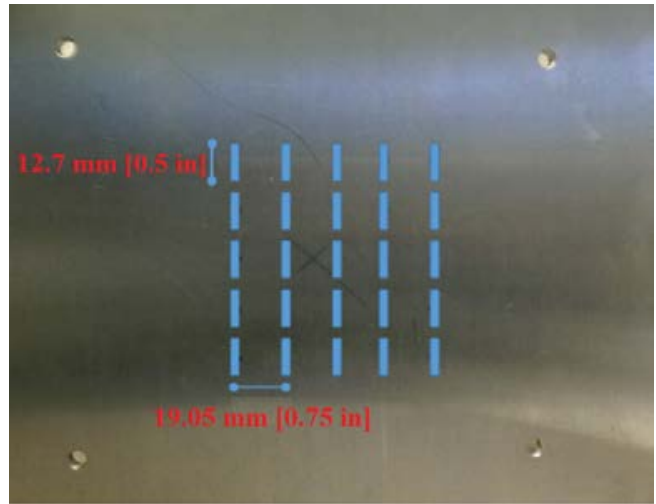

(a)

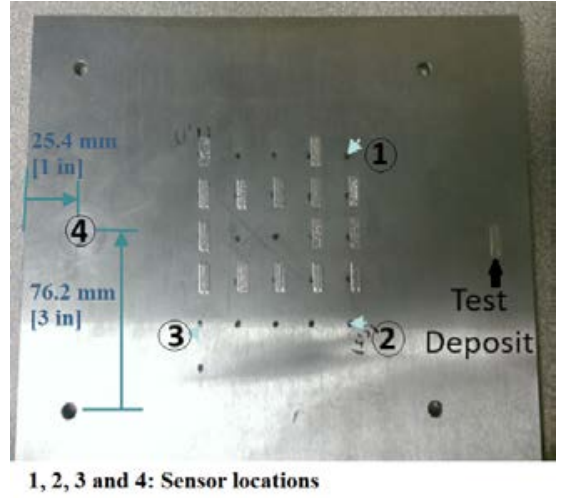

(b)

Figure 2. Direct Energy Deposition (DED) single layer deposition of Titanium 6Al-4V powder on steel build plate on $5 \times 5$ grid, (a) test grid and related dimensions, (b) location of the sensors related to the grid and on the build plate

A typical section of "RF" data record and corresponding spectrum are shown in Figure 3, which indicates that there are two main frequency bands where the majority of energy occurs. Based on this observation, the band of frequencies observed was divided into a low frequency band $(<800 \mathrm{kHz})$ and a high frequency band $(>800 \mathrm{kHz})$, and the dominant temporal and spectral features in each were investigated. 


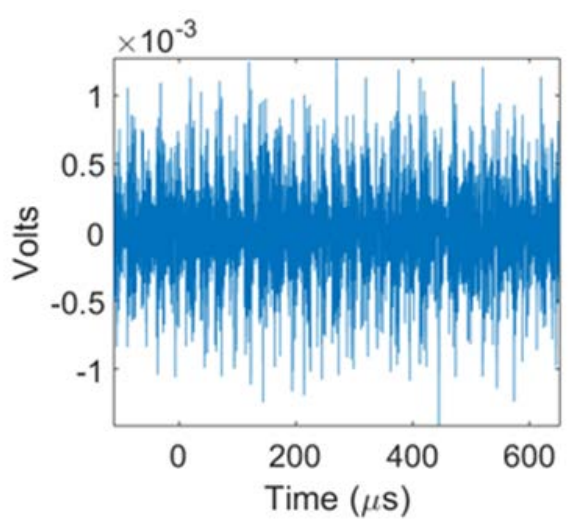

(a)

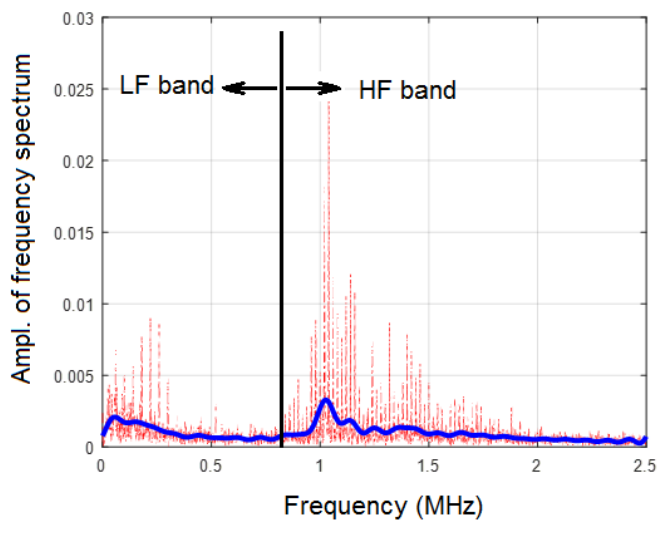

(b)

Figure 3. An example of the (a) original RF acoustic signal, and (b) frequency bands segments

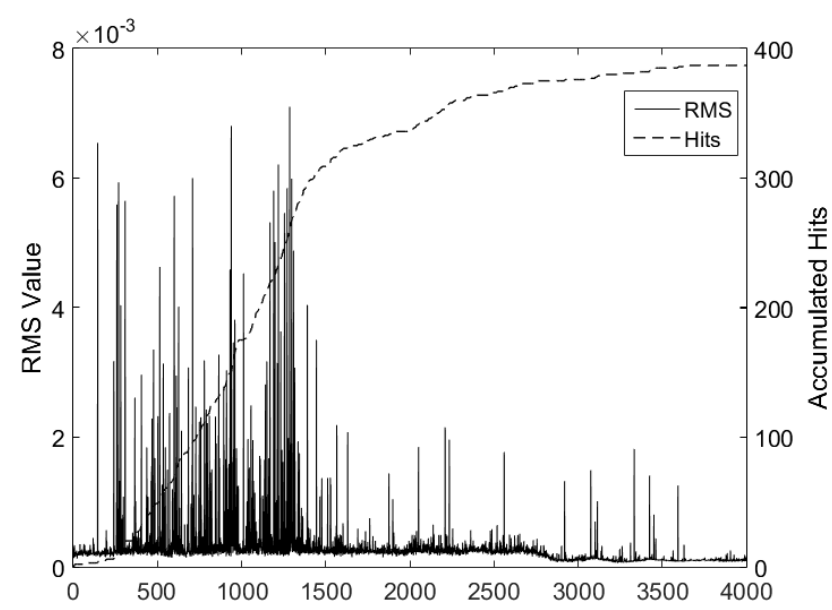

Figure 4. RMS noise levels for a normal build condition showing an underlying process noise and interspersed hits. An accumulated number of the hits over the course of the build is also shown as an indicator of material state

\section{Temporal metrics and analysis of acoustic signatures}

The signal processing employed consisted of band-pass filtering (150 kHz-2MHz) applied using a Kaiser order filter design and a convolution filter. Baseline subtraction was utilized to remove any signal drift and electronic noise using interpolated noise power spectra between intermittent baselines taken during testing (Boll), a common noise suppression technique in speech processing.

In acoustic emissions literature 'Hits' are defined as a signal that exceeds some predefined threshold and these are generally counted over time. They are also used to identify waveforms for further investigation and characterization. Hits were identified as waveforms with amplitudes exceeding 2 standard deviations from the mean $(\sim 95 \%$ confidence interval). The accumulation of hits over the course of a build under Normal conditions in depicted in Fig. 4. It can be observed that the hit total rises quickly during the build, and settles after build completion ( 2500 waveforms). Hits have been associated in previous work with formation of material defects including porosity and cracking ${ }^{16}$ and this can be used as a material state indicator.

Excluding waveforms identified as hits shows a marked decrease in RMS noise after build completion in Figure 5. Also displayed is the Baseline level RMS noise level which the Normal build condition approaches upon build completion. 
Accumulating temporal metrics and fitting with normal distributions, the scatter in the central tendency (mean) and standard deviation appear to follow consistent trends as seen in Figure. 6.

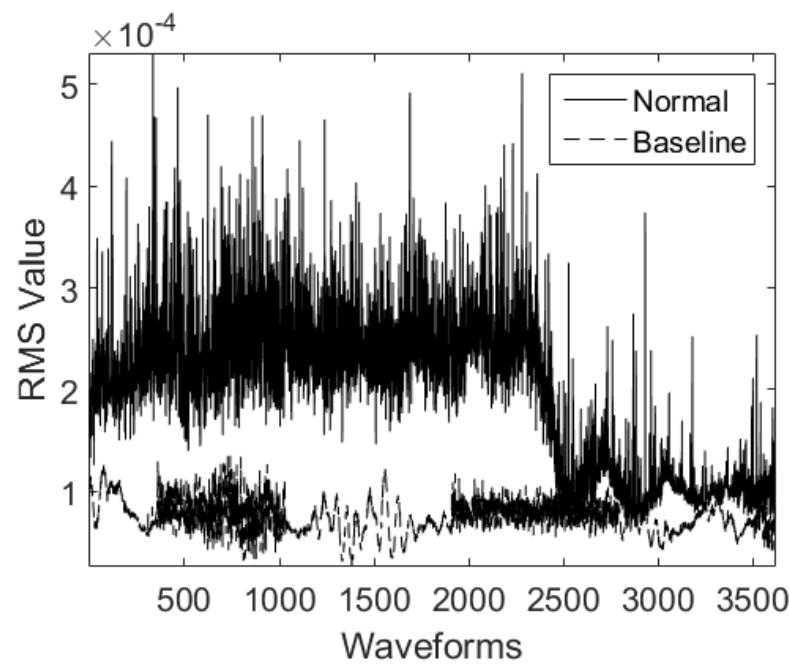

Figure 5. RMS noise values for the same Normal build condition depicted in Fig. 4 with waveforms associated with Hits removed to isolate process noise. A marked reduction in RMS noise levels is seen at approximately 2500

Waveforms coinciding with build completion and a return to Baseline RMS noise levels

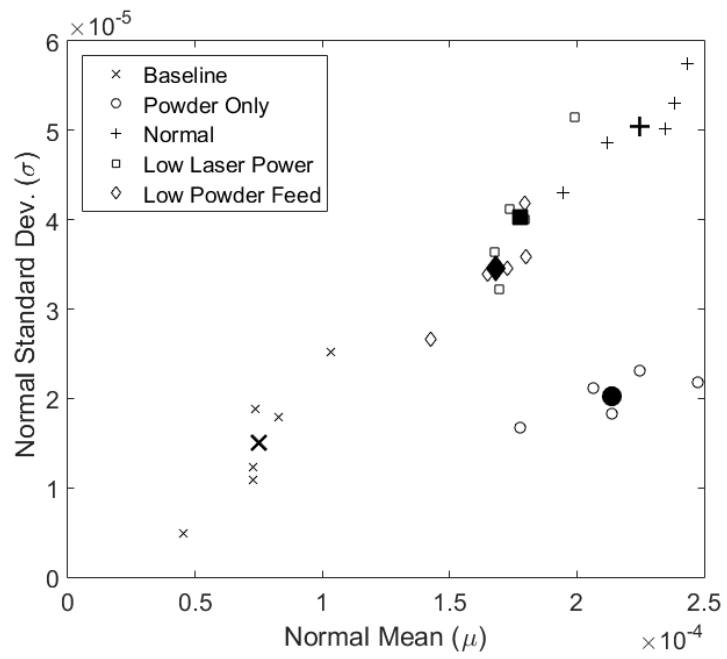

Figure 6. Clustering based on build conditions show consistency between builds of the same condition (same markers) and separation with other conditions (unique markers). Central tendencies of a fitted normal distribution to RMS noise level distributions tend to be elevated above baseline for all conditions, and with stronger deviations for builds entailing material deposition.

\section{Spectral metrics and analysis of acoustic signatures}

The use of frequency domain spectral features has proved to be particularly useful when there are a variety of different noise generation mechanisms in a system, such as in manufacturing machinery ${ }^{17}$, processing systems including boilers and heat exchangers, and in turbo-machinery fault diagnosis ${ }^{18}$. If defined appropriately, the frequency-related features and signatures for the acoustic signal are very effective in terms of "event" or source extraction and their use for discrimination and classification purposes. 
The method used for data analysis used here is based on feature extraction from the frequency response of the acoustic signature signals. Defined features in the spectral domain are listed in Table 1.

Table 1. Spectral features type and abbreviation

\begin{tabular}{|l|l|c|}
\hline Feature Number & \multicolumn{1}{|c|}{ Feature Description } & $\begin{array}{c}\text { Feature's } \\
\text { abbreviation }\end{array}$ \\
\hline Feature 1 & $\begin{array}{l}\text { peak amplitudes of the spectral data from the Fourier } \\
\text { Transform }\end{array}$ & PA \\
\hline Feature 2 & $\begin{array}{l}\text { Difference in peak amplitudes of the spectral data from } \\
\text { Fourier Transform for each condition compared to the one } \\
\text { for baseline condition }\end{array}$ & PAD \\
\hline Feature 3 & $\begin{array}{l}\text { Peak frequency of the spectral data from of Fourier } \\
\text { Transform }\end{array}$ & Pf \\
\hline Feature 4 & $\begin{array}{l}\text { Centroid amplitude of the spectral data from Fourier } \\
\text { Transform }\end{array}$ & CA \\
\hline Feature 5 & $\begin{array}{l}\text { Centroid frequency of the spectral data from Fourier } \\
\text { Transform }\end{array}$ & Cf \\
\hline
\end{tabular}

Clustering analysis is based on plotting the identified features in pairs and triples (2D and 3D respectively) to study the separation of variables and classification of process conditions for different build settings. The Clustering results for data from an acoustic piezoelectric sensor using centroid frequency $(C f)$ and centroid amplitude $(C A)$ of spectral data obtained using the Fourier Transform in low frequency and high frequency bands are shown in Figure 7. The classification of process conditions using three frequency domain features; centroid frequency $(C f)$ and centroid amplitude $(C A)$, and peak amplitude of frequency spectrum $(P A)$ of spectral data obtained using the Fourier transform for a single sensor at low and high frequency bands is shown in Figure. 8.

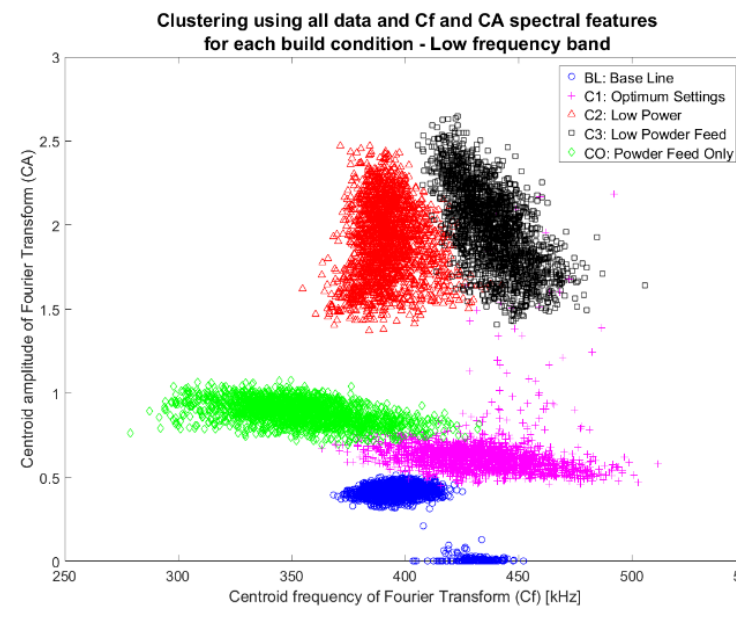

(a)

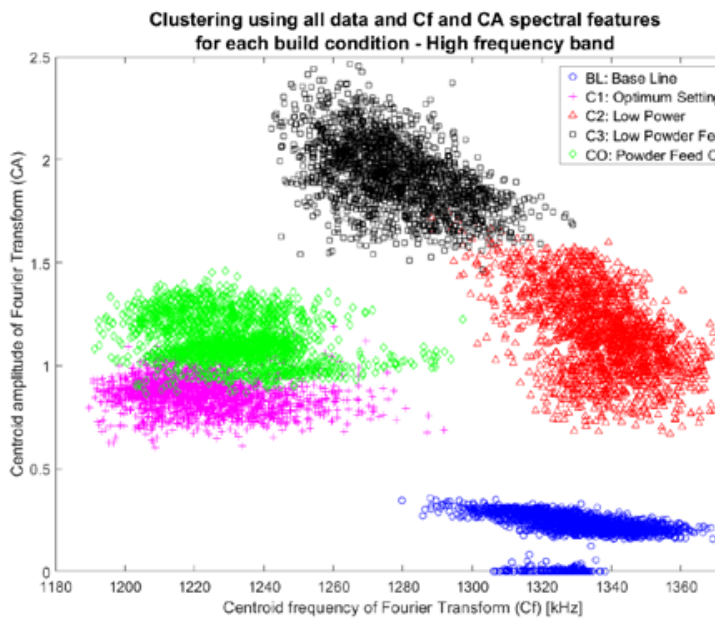

(b)

Figure 7. Clustering results for data from a single sensor using centroid frequency $(C f)$ and centroid amplitude $(C A)$ of spectral data obtained using the Fourier Transform in (a) low frequency and (b) high frequency bands 


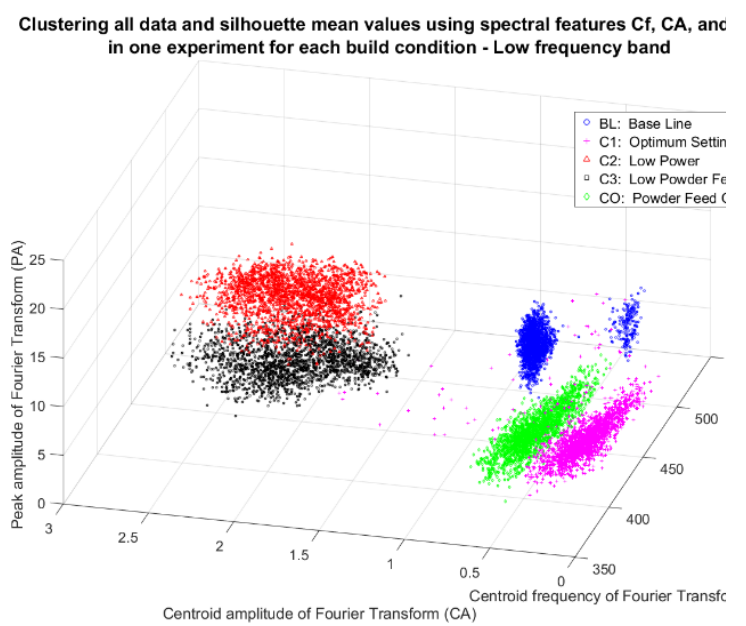

(a)

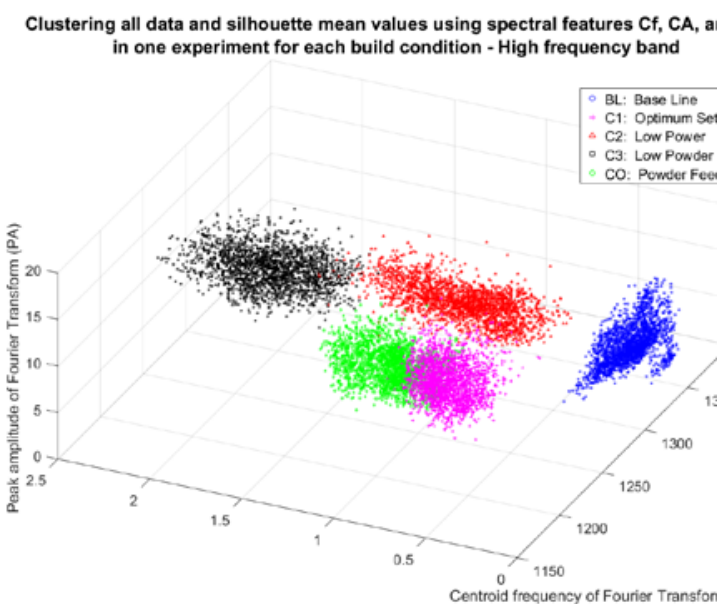

(b)

Figure 8. Classification of process conditions using three frequency domain features; peak amplitude of frequency spectrum $(P A)$, centroid frequency $(C f)$ and centroid amplitude $(C A)$ of spectral data obtained using the Fourier Transform for a single sensor at (a) low frequency and (b) high frequency bands

Distinct separations of clusters are seen in Figures 7 and 8 to correlate with the data from different process conditions. Combination of acoustic signatures in the frequency domain can provide closer and more effective data clustering for different process conditions.

\section{CONCLUSIONS}

A proof-of-concept study has investigated new approaches to process monitoring for additive manufacturing based on acoustic signatures. Various alternatives for signal processing, pattern recognition, and classification methods were applied to acoustic signals generated by an additive manufacturing process. It has been shown that acoustic signal characteristics can be used to classify process and system conditions. The acoustic signals were collected during the Direct Energy Deposition (DED) additive manufacturing process operated under different process conditions. A novel application of signal processing tools is used for the identification and use of metrics based on frequency spectral features in acoustic signals for the purpose of in-situ monitoring and characterization of conditions in an additive manufacturing process. A spectral feature-based clustering method was implemented to analyse the acoustic signals. Clustering plots for metrics in 2 and 3-D were used to facilitate the visualization of the groupings and condition discrimination. It is demonstrated that a passive acoustic monitoring approach and use of signal processing algorithms is effective at giving metrics that achieve clustering and separation of conditions based on multiple spectral features extracted from the original test data, and that these metrics do correlate with different AM system conditions.

Classification of different DED additive manufacturing process conditions exhibit successful clustering of large data sets. Evaluation of the identified features confirmed the consistency in process monitoring and data collection by all sensors, different locations on the build plate, and various process conditions. Results show that this novel approach using acoustic signal analysis can provide metrics based on acoustic signals (signatures) generated by the AM process, and classification of the signatures can be correlated with different process conditions.

Monitoring of the manufacturing process using acoustic signatures would appear to have the potential to give data which can help enable early detection of off-normal conditions, generation of faults in the process, and can be used for process optimization and control. 


\section{Acknowledgements}

This project was funded as an Industry-University Core Project by the Center for NDE (CNDE), Iowa State University, and the project was initiated while CNDE was a Phase III NSF Industry University Cooperate Research Center (IUCRC). Thanks go to Quad City Manufacturing Lab (QCML) who has provided access to and operation of the Direct Energy Deposition (DED) system for generation of the experimental data. This work was first reported at the $12^{\text {th }}$ ECNDT, Sweden, June 2018.

\section{References}

[1] Bond, L.J., Gray, J.N., Margetan, F., Utrata, D. and Anderson, I.E., "NDE for adding value to materials from metal powder processing". Proc. 2014 World Congress on Powder Metallurgy and Particulate Materials, (PM 2014) , 1944-59, (2014).

[2] Koester, L.W., Bond, L.J., Collins, P.C., Taheri H. and Bigelow, T.A., "Non-Destructive Evaluation of Additively Manufactured Metallic Parts". Metals Handbook Vol. 17, ASM International, 544-552 (2018).

[3] Koester, L.W., Taheri, H., Bigelow, T.A., Collins, P.C. and Bond, L.J., "Nondestructive testing for parts fabricated using additive manufacturing," Materials Evaluation. 76 (4) 514-524 (2018).

[4] Koester, L., Roberts, R. A., Barnard, D. J., Chakrapani, S., Singh, S., Hogan, R. and Bond, L. J., "NDE of additively manufactured components with embedded defects (reference standards) using conventional and advanced ultrasonic methods." AIP Conference Proceedings 1806, paper. 140006 (2017).

[5] Taheri, H., Koester, L., Bigelow, T. and Bond, L.J., " Finite element simulation and experimental verification of ultrasonic non-destructive inspection in additively manufactured materials." Proc. 44th Annual Review of Progress in Quantitative Nondestructive Evaluation, American Institute of Physics (AIP), Conference Proceedings \# 1949, 020011 (2018).

[6] Taheri, H., Shoaib, M.R.M., Koester, L.W., Bigelow, T.A., Collins P.C. and Bond, L.J., "Powder based additive manufacturing - A review of types of defects, generation mechanisms, detection, property evaluation and metrology". Int. J Additive Subtractive Material Manufacturing 1 (2) 172-209 ( 2017).

[7] Everton, S.K., Hirsch, M., Stravroulakis, P., Leach R.K. and Clare, A.T., "Review of in-situ process monitoring and in-situ metrology for metal additive manufacturing. Mater. Des. 95, $431-45$ (2016).

[8] Farson, D.F. and Kim, K.R., "Generation of optical and acoustic emissions in laser weld plumes". J. Appl. Phys. 85

(3) $1329-1336$ (1999).

[9] Krauss, H., Zeugner T. and Zaeh, M.F., "Thermographic process monitoring in powder based additive manufacturing". AIP Conf. Proc. 1650, 177-83 (2015).

[10] Rieder, H., Dillhöfer, A., Spies, M., Bamberg, J. and Hess, T., "Online Monitoring of Additive Manufacturing Processes Using Ultrasound", Proc. $11^{\text {th }}$ Eur. Conf. Non-Destructive Test. (ECNDT 2014), 2194-2201 (2014).

[11] Miller, M., Mi, B., Kita, A. and Ume, I.C., "Development of automated real-time data acquisition system for robotic weld quality monitoring". Mechatronics. 12 (9-10) 1259-1269 (2002).

[12] Taheri, H., Koester, L.W., Bigelow, T.A. and Bond, L.J., "Thermoelastic finite element modeling of laser generated ultrasound in additive manufacturing materials". Proc. ASNT Annual Conference. 188-198 (2017).

[13] Koester, L.W., Taheri, H., Bigelow, T.A., Bond, L. J. and Faierson, E.J., "In-situ acoustic signature monitoring in additive manufacturing processes." Proc. 44th Annual Review of Progress in Quantitative Nondestructive Evaluation, American Institute of Physics (AIP), Conference Proceedings \# 1949 (1), 020006 (2018)

[14] Taheri, H., Koester, L.W., Bigelow, T.A., Faierson, E. and Bond, L.J., "In-situ Process Monitoring of Additive Manufacturing using Clustering of Spectral Features for Acoustic Signals”. J. Manuf. Sci. Eng. - Trans. ASME. (inpress) 2019.

[15] Gold, S.A. and Spears, T.G., "Acoustic monitoring method for additive manufacturing processes". Patent US 20170146488 A1, (2017).

[16] Haythem Gaja, H. and Liou, F., "Defects monitoring of laser metal deposition using acoustic emission sensor," The International Journal of Advanced Manufacturing Technology, 90 (1-4) 561-574|(2017).

[17] Hassaan, G.A., "Frequency Spectrum Filtering For Machinery Fault Diagnostics". Int. J. Sci. Technol. Res. 3 (8) 200-203 (2014).

[18] Al-Hashmi, S.A., "Spectrum analysis of acoustic signals for cavitation detection". Proc. IEEE Symposium on Industrial Electronics and Applications (ISIEA2012), 321-5 (2012). 